\title{
Inpatient patient safety events in vulnerable populations: a retrospective cohort study
}

\author{
Lucy B Schulson (D) , 1,2 Victor Novack, ${ }^{3,4}$ Patricia H Folcarelli, ${ }^{5,6}$ \\ Jennifer P Stevens, ${ }^{3,7}$ Bruce E Landon ${ }^{3,6,8}$
}

\begin{abstract}
- Additional material is published online only. To view, please visit the journal online (http://dx.doi.org/10.1136/ bmjas-2020-011920).
\end{abstract}

For numbered affiliations see end of article.

\section{Correspondence to}

Dr Lucy B Schulson, RAND Corp Boston office, Boston MA 02116, USA; schulson@rand.org

Received 2 July 2020 Revised 28 September 2020 Accepted 2 October 2020 Published Online First 26 October 2020

\section{Linked}

- http://dx.doi.org/10.1136/ bmjgs-2020-012599

\section{Check for updates}

(c) Author(s) (or their employer(s)) 2021. No commercial re-use. See rights and permissions. Published by BMJ.

To cite: Schulson $L B$, Novack V, Folcarelli PH, et al. BMJ Qual Saf

2021:30:372-379.

\begin{abstract}
Background Widespread attention to structural racism has heightened interest in disparities in the quality of care delivered to racial/ethnic minorities and other vulnerable populations. These groups may also be at increased risk of patient safety events.

Objective To examine differences in inpatient patient safety events for vulnerable populations defined by race/ ethnicity, insurance status and limited English proficiency (LEP).

Design Retrospective cohort study.

Setting Single tertiary care academic medical centre.

Participants Inpatient admissions of those aged $\geq 18$ years from 1 October 2014 to 31 December 2018.

Measurements Primary exposures of interest were self-identified race/ethnicity, Medicaid insurance/ uninsured and LEP. The primary outcome of interest was the total number of patient safety events, defined as any event identified by a modified version of the Institute for Healthcare Improvement global trigger tool that automatically identifies patient safety events ('automated') from the electronic record or by the hospital-wide voluntary provider reporting system ('voluntary'). Negative binomial models were used to adjust for demographic and clinical factors. We also stratified results by automated and voluntary.

Results We studied 141877 hospitalisations, of which $13.6 \%$ had any patient safety event. In adjusted analyses, Asian race/ethnicity was associated with a lower event rate (incident rate ratio (IRR) $0.89,95 \% \mathrm{Cl} 0.83$ to 0.96); LEP patients had a lower risk of any patient safety event and voluntary events (IRR $0.91,95 \% \mathrm{Cl} 0.87$ to 0.96; IRR $0.89,95 \% \mathrm{Cl} 0.85$ to 0.94). Asian and Latino race/ethnicity were also associated with a lower rate of voluntary events but no difference in risk of automated events. Black race was associated with an increased risk of automated events (IRR 1.11,95\% Cl 1.03 to 1.20).

Limitations This is a single centre study.

Conclusions A commonly used method for monitoring patient safety problems, namely voluntary incident reporting, may underdetect safety events in vulnerable populations.
\end{abstract}

\section{INTRODUCTION}

The current social political climate in the USA and globally has led to heightened attention to health disparities. This has led to a re-examining of how health systems may contribute to these disparities. Patient safety events are 'events, incident[s], or condition $[\mathrm{s}]$ that could have resulted or did result in harm to a patient'. Such events are an important cause of morbidity and mortality in the USA and vulnerable population, including racial and ethnic minorities, those on public insurance or lacking insurance and those with limited English proficiency (LEP) may be at increased risk. Multiple potential mechanisms could contribute to such increased risk. For example, these groups may lack the social capital to advocate for themselves in the way that other populations can and this issue might be compounded by lower health literacy. ${ }^{2-4}$ Those with LEP may be at particular risk for patient safety events due to communication barriers and inconsistent use of interpreters. $^{5-7}$ Finally, racial and ethnic minorities are more likely to experience discrimination, and it is possible that bias in their care could contribute to patient safety events. ${ }^{8}$

Because of both the fast-paced nature and intensity of hospital care, hospitalised patients are at particular risk for patient safety events, and vulnerable patient populations might be at particularly increased risk. However, the current systems used to identify patient safety events may not adequately identify events in vulnerable patient populations. The principal approaches to identifying patient safety events include voluntary reporting systems and automated systems that rely on the electronic medical record, and each of these suffers from limitations. For instance, although voluntary reporting systems can identify near misses and other more nuanced events, they suffer from under-reporting and are subject to judgement and personal biases, which may disproportionately impact reporting for vulnerable populations. ${ }^{9-12}$ 
In contrast, systems that rely on automatically collected data, including electronic-medical recordbased systems, such as one based on the Institute for Healthcare Improvement (IHI)'s global trigger tool, may be more objective than a voluntary system, and may capture data in a more reliable manner. However, because such a tool depends on data being captured in the electronic medical record, they also are inherently limited in scope. ${ }^{913}$

In this study, we examined the extent to which race/ ethnicity, insurance status and LEP are associated with risk for patient safety events in adult inpatients. We used two complementary methods to identify events including a voluntary hospital-wide incident reporting system and an automated system based on a modified version of the IHI Global Trigger tool that leverages data in the electronic medical record to automatically identify safety events. ${ }^{14}$

\section{METHODS}

\section{Study sample}

We conducted a retrospective cohort study of patients aged $\geq 18$ years admitted to a tertiary care, urban academic medical centre under observation or inpatient status between 1 October 2014 and 31 December 2018, excluding those admitted with a psychiatric diagnosis, transferred to another hospital or who lacked a primary diagnosis $(<0.01 \%)$.

\section{Exposures of interest}

To identify vulnerable populations, we relied on three complementary approaches. To identify patients with LEP, we relied on the administrative records of the hospital. When initially registering at the hospital, patients are asked their preferred language for the purposes of identifying those who might need interpreters. We categorised patients as LEP if a language other than English was their preferred language. The small number of individuals for whom language was unknown or missing also were categorised as LEP $(\mathrm{n}=52,0.04 \%)$. To identify those from racial or ethnic minorities, we similarly used patient's self-reported race and ethnicity when registering at the hospital. We categorised race/ethnicity as White, Black, Latino/ Hispanic (Latino), Asian and other/unknown/missing (other). Finally, as a proxy for socioeconomic status, we identified primary insurance type which we categorised as commercial/Medicare/other (other) and Medicaid/safety net/self-pay (Medicaid), focusing on the Medicaid population in our primary analyses. Patients with both Medicare and Medicaid were categorised as having Medicaid.

For the LEP population, we further classified them according to the most common preferred languages: Spanish, Chinese (Mandarin and Cantonese), Cape Verdean or Russian. All other languages, including those with missing or unknown language, were categorised as 'other'.
Table 1 Types of patient safety events

\begin{tabular}{|c|c|}
\hline Event type & Example/Source \\
\hline \multicolumn{2}{|l|}{ Voluntary reported patient safety events } \\
\hline Medication/Fluid event & Adverse reaction, missed dose \\
\hline Skin/Tissue injury & Pressure ulcer \\
\hline Diagnosis/Treatment & Delay in diagnosis \\
\hline Radiology & abnormal results not reported \\
\hline Surgery/Procedure & Count discrepancy \\
\hline Lab/Specimen & $\begin{array}{l}\text { Documentation wrong patientWrong } \\
\text { patient identified }\end{array}$ \\
\hline Feedback from patients & Grievance \\
\hline Falls & From bed \\
\hline $\begin{array}{l}\text { Communication/Coordination/Handoff } \\
\text { issues: }\end{array}$ & Inappropriate discharge \\
\hline Infection events & Isolation inadequate \\
\hline Patient ID/Documentation/Consent & Consent issue \\
\hline Intravenous access issue & Disconnected, infiltration \\
\hline Code blue & Cardiac or pulmonary arrest \\
\hline Code purple & Psychiatric emergency \\
\hline Respect and dignity & Emotional harm to patient \\
\hline Professional conduct & Discrimination \\
\hline Safety/Security & Abduction \\
\hline Blood product & Contraindication to administration \\
\hline Maternal/Childbirth & Maternal death \\
\hline Nutrition & Patient given food when NPO \\
\hline Restraints & Injury-restraint related \\
\hline \multicolumn{2}{|l|}{ Automated patient safety events } \\
\hline Clostridium difficile infection & Microbiology \\
\hline Bloodstream infection & Microbiology \\
\hline Unrecognised bleeding & Lab \\
\hline PTT $>100$ while on heparin & Lab and pharmacy \\
\hline INR $>6$ while on warfarin & Lab and pharmacy \\
\hline Glucose $<50$ while on insulin & Lab and pharmacy \\
\hline Acute renal injury & Lab \\
\hline Administration of vitamin $\mathrm{K}$ & Pharmacy \\
\hline Administration of naloxone & Pharmacy \\
\hline
\end{tabular}

${ }^{*} \sim 10 \%$ of the orders of naloxone were dispensed but not administered.

INR, international normalised ratio; PTT, partial thromboplastin time.

\section{Outcomes of interest}

We identified patient safety events using two complementary identification systems.

Voluntary events were identified using a commercially available safety reporting system (RLDatix), which is an online system where employees are encouraged to report a patient safety event or near miss. ${ }^{15}$ When reporting an event, the reporter determines the relevant category, which includes medication-related, skin/tissue injuries, diagnosis/treatment-related, radiology, surgery/procedure-related, lab/specimen, feedback from patients, falls, communication/coordination/handoffs, infection, patient ID/documentation/ consent-related, intravenous access issue, code blue (cardiac arrest), code purple (psychiatric emergency), respect and dignity, professional conduct, security/ safety, blood products, maternal/childbirth, nutrition and use of restraints (table 1). 
Automated patient safety events were identified using the hospital's electronic record data and consisted of a subset of measures from the IHI global trigger tool suitable for automated capture. Events included Clostridium difficile infection following antibiotics, healthcare-associated bloodstream infection, unrecognised bleeding, partial thromboplastin time (PTT) $>100$ on heparin, international normalised ratio $>6$ on warfarin, glucose $<50$ on insulin, acute renal injury while hospitalised, inpatient administration of vitamin $\mathrm{K}$ and inpatient dispensing of naloxone (table 1). The traditional IHI global trigger tool relies on the ' $20 \mathrm{~min}$ rule' where records are reviewed by an individual for no longer than 20 min. ${ }^{14}$ The modified version does not depend on chart review by an individual and unlike the IHI global trigger tool, data are pulled in real time which allows for the use of logical statements to more accurately identify events. ${ }^{16}$ Algorithms were created to flag the data from the electronic medical record and create the logic (eg, the PTT >100 flagging if the patient was on heparin). By construction, these measures almost universally indicate an actual adverse event (even if there were no clinical consequences), thus resulting in a higher positive predictive value than seen with the traditional global trigger tool. This latter method for identifying patient safety events was validated as part of quality improvement efforts in the study institution. A research nurse reviewed the records of 237 consecutive intensive care unit (ICU) admissions and found a sensitivity of $100 \%$, a specificity of $99.8 \%$ and a positive predictive value of $93 \%$ of this method to identify harms. ${ }^{16}$

\section{Control variables}

We ascertained age (categorised as 18-29, 31-40, $41-50,51-64$ and $\geq 65$ years) and sex from the medical record, from which we also identified marital status (married/partner 'married' or divorced/separated/widow/single/unknown or missing 'other') as a marker of social support as this may influence a patient's risk of an event. For each admission, we also identified year of admission, length of stay and clinical service. Using billing codes, we identified the primary diagnosis, which we then classified using Clinical Classification Software (CCS) for International Classification of Diseases (ICD)-9 and ICD-10. ${ }^{17}$ We used the Elixhauser comorbidities to calculate a clinical risk score based on coded comorbid conditions from electronic billing data. ${ }^{18}$ We also identified those patients who spent part of their stay in the ICU to additionally account for acuity.

\section{Statistical analyses}

We performed unadjusted comparisons using $\chi^{2}$ and Wilcoxon signed-rank test as appropriate to identify characteristics associated with at least one patient safety event, at least one voluntary reported safety event and at least one automated safety event. We then estimated negative binomial regression models with generalised estimation equations to account for clustering of multiple hospitalisations per patient with compound symmetry covariance matrix adjusting for age, sex, race, LEP status, insurance type (Medicaid/ uninsured 'Medicaid' vs other), CCS code, clinical risk score, year of admission, primary service at discharge and length of stay. In our primary analyses, insurance, race and LEP were entered individually (along with the other control variables) due to concern for the interconnectedness of these characteristics, but we also ran analyses where all of these variables were included simultaneously (online supplemental appendix tables 1 and 2). In our main analyses, our primary outcome of interest was total number of patient safety events, which combined the voluntary and automated events. In secondary analyses, we also examined total voluntary and automated events individually and report these separately. We additionally examined results stratified by language (online supplemental appendix table 3). All statistical tests were two-tailed and $\alpha$ was set at 0.05 . We calculated incident rate ratios (IRRs) with 95\% CIs. All analyses were performed using SAS (V.9.4).

\section{RESULTS}

We studied 141877 hospitalisations over the time period of interest. Race/ethnicity was missing for $11.4 \%$ of the sample $(n=16185)$. The average age of the admitted patients was 58 (standard deviation \pm 19.3 ) and $58.3 \%$ were female. The sample was $10.1 \%$ LEP, $12.7 \%$ Black, 4.4\% Latino and 5.2\% Asian. Those with Medicaid made up $16.4 \%$ of the sample. The median length of stay was 3 days (IQR 2-6) and the median clinical risk score (comorbidity score) was 0.86 (IQR 0.68-2.1). Across these hospitalisations, 19280 $(13.6 \%)$ resulted in at least one patient safety event, including $15480(10.6 \%)$ with at least one voluntary reported patient safety event and 6459 (4.6\%) with at least one automated patient safety event (table 2).

\section{Unadjusted results}

In unadjusted analyses, older patients and those who were male were more likely to experience one or more patient safety events $(55.4 \%$ of those with an event were age $\geq 65$ years vs $42.1 \%$ in sample, $p<0.0001$ and $49.4 \%$ of those with an event were males vs $41.7 \%$ in sample, $\mathrm{p}<0.0001)$. Those with a patient safety event also had longer lengths of stay (8 days IQR 4-15 vs 3 days IQR 2-6 in sample, $\mathrm{p}<0.0001$ ), and higher clinical risk scores (median 2.0 IQR 0.85-5.9 vs median 0.86 IQR $0.68-2.1$ in sample, $\mathrm{p}<0.0001$ ). White and Black patients were more likely than other race/ethnicities to have at least one patient safety event $(67.5 \%$ of those with an event were White vs $64.3 \%$ in sample, $13.1 \%$ of those with an event were Black vs $12.7 \%$ in sample, $\mathrm{p}<0.0001)$. Patients with Medicaid were less likely to have at least one safety event compared 
Table 2 Sample characteristics and unadjusted rates of patient safety events per hospitalisation

\begin{tabular}{|c|c|c|c|c|c|c|c|}
\hline Variable & $\begin{array}{l}\text { Overall } \\
\text { sample }\end{array}$ & $\begin{array}{l}\text { Patient safety } \\
\text { event }\end{array}$ & $P$ value ${ }^{*} \dagger$ & $\begin{array}{l}\text { Voluntary } \\
\text { patient safety } \\
\text { event }\end{array}$ & P value* $\ddagger$ & $\begin{array}{l}\text { Automated patient } \\
\text { safety event }\end{array}$ & P value ${ }^{*} \S$ \\
\hline & $n=141877$ & $n=19280$ & & $n=15048$ & & $n=6459$ & \\
\hline & & $13.6 \%$ & & $10.6 \%$ & & $4.6 \%$ & \\
\hline \multicolumn{8}{|l|}{ Sex } \\
\hline Female & $58.3 \%$ & $50.6 \%$ & $<0.0001$ & $51.0 \%$ & $<0.0001$ & $47.7 \%$ & $<0.0001$ \\
\hline Age (years) & & & $<0.0001$ & & $<0.0001$ & & $<0.0001$ \\
\hline $18-29$ & $9.4 \%$ & $4.6 \%$ & & $5.1 \%$ & & $2.5 \%$ & \\
\hline $31-40$ & $15.8 \%$ & $7.4 \%$ & & $8.2 \%$ & & $4.6 \%$ & \\
\hline $41-50$ & $9.2 \%$ & $8.0 \%$ & & $8.0 \%$ & & $8.1 \%$ & \\
\hline $51-64$ & $23.6 \%$ & $24.6 \%$ & & $23.9 \%$ & & $27.3 \%$ & \\
\hline$\geq 65$ & $42.1 \%$ & $55.4 \%$ & & $54.8 \%$ & & $57.5 \%$ & \\
\hline Race/Ethnicity & & & $<0.0001$ & & $<0.0001$ & & $<0.0001$ \\
\hline White & $64.3 \%$ & $67.5 \%$ & & $67.7 \%$ & & $66.3 \%$ & \\
\hline Black & $12.7 \%$ & $13.1 \%$ & & $12.7 \%$ & & $14.1 \%$ & \\
\hline Latino & $4.4 \%$ & $3.8 \%$ & & $3.7 \%$ & & $4.2 \%$ & \\
\hline Asian & $5.2 \%$ & $3.4 \%$ & & $3.5 \%$ & & $3.2 \%$ & \\
\hline Other & $13.3 \%$ & $12.3 \%$ & & $12.4 \%$ & & $12.3 \%$ & \\
\hline LEP & $10.1 \%$ & $10.1 \%$ & 0.97 & $10.1 \%$ & 0.87 & $10.6 \%$ & 0.14 \\
\hline Language & & & $<0.0001$ & & $<0.0001$ & & $<0.0001$ \\
\hline English & $89.9 \%$ & $89.9 \%$ & & $89.9 \%$ & & $89.4 \%$ & $<0.0001$ \\
\hline Spanish & $2.8 \%$ & $3.1 \%$ & & $2.9 \%$ & & $3.6 \%$ & \\
\hline Chinese & $2.2 \%$ & $1.4 \%$ & & $1.5 \%$ & & $1.2 \%$ & \\
\hline Russian & $1.1 \%$ & $1.4 \%$ & & $1.5 \%$ & & $1.4 \%$ & \\
\hline Cape Verdean & $0.8 \%$ & $0.8 \%$ & & $0.8 \%$ & & $1.0 \%$ & \\
\hline Other & $3.1 \%$ & $3.4 \%$ & & $3.4 \%$ & & $3.5 \%$ & \\
\hline Marital status & & & $<0.0001$ & & $<0.0001$ & & $<0.0001$ \\
\hline Married/Life partner & $49.0 \%$ & $43.4 \%$ & & $42.9 \%$ & & $43.9 \%$ & \\
\hline Other & $51.0 \%$ & $56.6 \%$ & & $57.1 \%$ & & $56.1 \%$ & \\
\hline Insurance & & & $<0.0001$ & & $<0.0001$ & & $<0.0001$ \\
\hline Other§ & $83.6 \%$ & $88.2 \%$ & & $90.8 \%$ & & $96.2 \%$ & \\
\hline Medicaid & $16.4 \%$ & $11.8 \%$ & & $9.3 \%$ & & $3.8 \%$ & \\
\hline Length of stay & 3 (2 to 6) & $8(4$ to 15$)$ & $<0.0001$ & $7(4$ to 15$)$ & $<0.0001$ & 11 (6 to 21) & \\
\hline Service & & & $<0.0001$ & & $<0.0001$ & & $<0.0001$ \\
\hline Medicine & $45.7 \%$ & $58.4 \%$ & & $56.4 \%$ & & $64.4 \%$ & \\
\hline Surgical & $31.6 \%$ & $31.5 \%$ & & $32.0 \%$ & & $31.1 \%$ & \\
\hline Obstetrics and gynaecology & $18.4 \%$ & $6.0 \%$ & & $7.1 \%$ & & $1.7 \%$ & \\
\hline Neurology & $4.3 \%$ & $4.1 \%$ & & $4.5 \%$ & & $2.8 \%$ & \\
\hline Primary diagnosis & & & $<0.0001$ & & $<0.0001$ & & $<0.0001$ \\
\hline Pregnancy related & $16.8 \%$ & $5.1 \%$ & & $6.2 \%$ & & $0.9 \%$ & \\
\hline Circulatory system & $18.6 \%$ & $23.5 \%$ & & $20.6 \%$ & & $32.3 \%$ & \\
\hline Digestive system & $11.0 \%$ & $10.9 \%$ & & $10.1 \%$ & & $12.2 \%$ & \\
\hline $\begin{array}{l}\text { Musculoskeletal/Connective } \\
\text { tissue }\end{array}$ & $6.2 \%$ & $4.6 \%$ & & $5.2 \%$ & & $2.3 \%$ & \\
\hline Pulmonary & $4.9 \%$ & $5.3 \%$ & & $5.5 \%$ & & $4.6 \%$ & \\
\hline Injury and poisoning & $11.8 \%$ & $15.2 \%$ & & $15.5 \%$ & & $14.8 \%$ & \\
\hline Haematology-oncology & $11.2 \%$ & $11.9 \%$ & & $12.2 \%$ & & $11.1 \%$ & \\
\hline Other & $19.5 \%$ & $23.7 \%$ & & $24.7 \%$ & & $21.9 \%$ & \\
\hline Clinical risk score ${ }^{* *}$ & $\begin{array}{l}0.86(0.68 \text { to } \\
2.1)\end{array}$ & $2.0(0.85$ to 5.9$)$ & $<0.0001$ & $2.0(0.84$ to 5.9$)$ & $<0.0001$ & 3.1 (1.1 to 8.9$)$ & \\
\hline Year & & & $<0.0001$ & & $<0.0001$ & & $<0.0001$ \\
\hline 2014 & $5.6 \%$ & $5.2 \%$ & & $5.1 \%$ & & $5.5 \%$ & \\
\hline 2015 & $23.2 \%$ & $21.9 \%$ & & $22.1 \%$ & & $21.1 \%$ & \\
\hline 2016 & $23.6 \%$ & $23.5 \%$ & & $24.0 \%$ & & $22.3 \%$ & \\
\hline 2017 & $23.9 \%$ & $24.4 \%$ & & $23.9 \%$ & & $25.9 \%$ & \\
\hline
\end{tabular}


Table 2 Continued

\begin{tabular}{|c|c|c|c|c|c|c|c|}
\hline Variable & $\begin{array}{l}\text { Overall } \\
\text { sample }\end{array}$ & $\begin{array}{l}\text { Patient safety } \\
\text { event }\end{array}$ & P value ${ }^{*} t$ & $\begin{array}{l}\text { Voluntary } \\
\text { patient safety } \\
\text { event }\end{array}$ & P value* $\ddagger$ & $\begin{array}{l}\text { Automated patient } \\
\text { safety event }\end{array}$ & P value ${ }^{*} \S$ \\
\hline 2018 & $23.7 \%$ & $24.9 \%$ & & $24.9 \%$ & & $25.3 \%$ & \\
\hline
\end{tabular}

${ }^{*} P$ values significant at $\alpha<0.05$.

†Compared with those without a patient safety event, column percent.

‡Compared with those without a voluntary reported patient safety event, column per cent.

$\S$ Combined: commercial, Medicare, other.

TMedicaid or uninsured.

${ }^{* *}$ Risk of death.

††Compared with those without an automatically reported patient safety event, column per cent.

$\ddagger \ddagger$ Based on clinical classification software.

LEP, limited English proficiency:;

with other types of insurance $(11.8 \%$ of those with an event had Medicaid insurance vs $16.4 \%$ in sample, $\mathrm{p}<0.0001$ ) (table 2).

In stratified analyses, White patients were more likely to have a voluntary patient safety event $(67.7 \%$ of those with an event vs $64.3 \%$ in sample, $p<0.0001$ ), and Latino and Asian patients were less likely $(3.7 \%$ of those with an event vs $4.4 \%$ in sample for Latino patients and $3.5 \%$ of those with an event vs $5.2 \%$ in sample for Asian patients, $\mathrm{p}<0.0001$ ). Black patients were more likely to have an automated patient safety event $(14.1 \%$ of those with an event vs $12.7 \%$ in sample, $\mathrm{p}<0.0001)$.

Patients with Medicaid were less likely than other insurance groups to have both voluntary and automated events $9.3 \%$ of voluntary reported events, $3.8 \%$ of automated events vs $16.4 \%$ in sample, all $\mathrm{p}<0.0001)$. There was no increased risk for either type of patient safety event for LEP patients compared with English proficient patients (table 2).

\section{Adjusted results overall}

In adjusted analyses where each predictor of interest (eg, race/ethnicity, LEP, Medicaid insurance) was entered individually, those with Asian race/ethnicity had significantly lower risk of experiencing a patient safety event (IRR $0.89,95 \%$ CI 0.83 to 0.96 ) and those with LEP had a significantly lower risk of a patient safety event (IRR $0.91,95 \%$ CI 0.87 to 0.96 ). There was no significant difference for other race/ ethnicities. Medicaid insurance was not significantly associated with increased risk for patient safety events (IRR $0.98,95 \%$ CI 0.94 to 1.02 ) (figure 1). Findings were attenuated when all three predictors of interest were included in the same model because of collinearity (online supplemental appendix table 1).

\section{Adjusted results stratified by reporting system}

\section{Voluntary events}

Latino and Asian patients were less likely to experience voluntary events (IRR $0.90,95 \%$ CI 0.83 to 0.98 and IRR $0.87,95 \%$ CI 0.80 to 0.96 , respectively) as were LEP patients (IRR $0.89,95 \%$ CI 0.85 to 0.94 ) (figure 2, online supplemental appendix table 2).
Medicaid insurance was not associated with a significant difference in risk for voluntary reported events.

\section{Automated events}

In contrast, Black patients were the only group that were more likely to experience an automated event (IRR 1.11, 95\% CI 1.03 to 1.20), although the event risk of Latino patients was of borderline significance (IRR $1.06,95 \%$ CI 0.93 to 1.20 ). LEP status and Medicaid insurance were not associated with a significant difference in risk for automated events (figure 2, online supplemental appendix table 2).

\section{Subanalyses by language}

Finally, analyses examining the largest groups of LEP patients demonstrated significant diversity within this group. For instance, Chinese speakers were less likely to have any patient safety event (IRR $0.84,95 \%$ CI 0.75 to 0.94 ) as well as a voluntary reported event (IRR 0.85 , 95\% CI 0.74 to 0.97). Spanish speakers were also less likely to have a voluntary reported

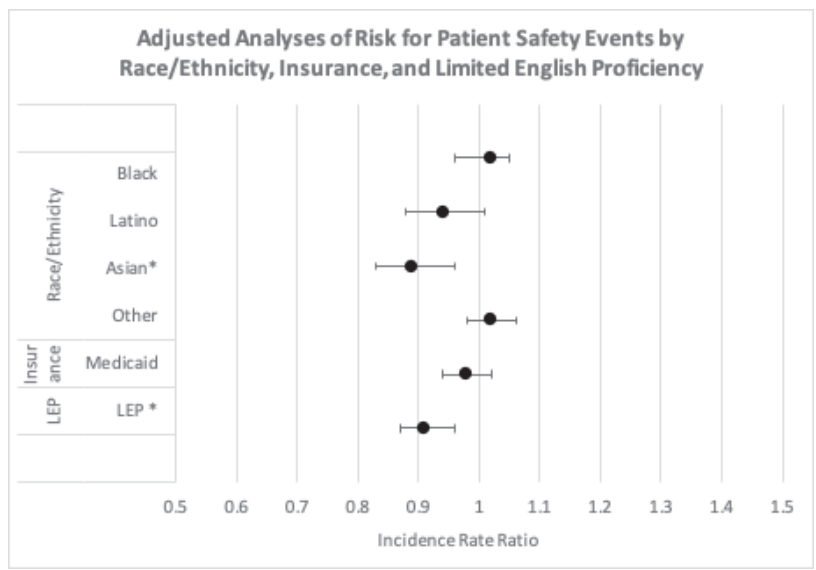

Figure 1 Adjusted analyses of risk for patient safety events by race/ ethnicity, insurance and limited English proficiency. LEP, limited English proficient; IRR, incident rate ratio. Adjusting for age, sex, marital status, primary diagnosis, clinical risk score, service at discharge, intensive care stay, length of stay and year of admission. Generalised estimation equations included to account for clustering for multiple admissions per patient. Race/Ethnicity, insurance and LEP are entered individually. ${ }^{*} \mathrm{P}<0.05$. 


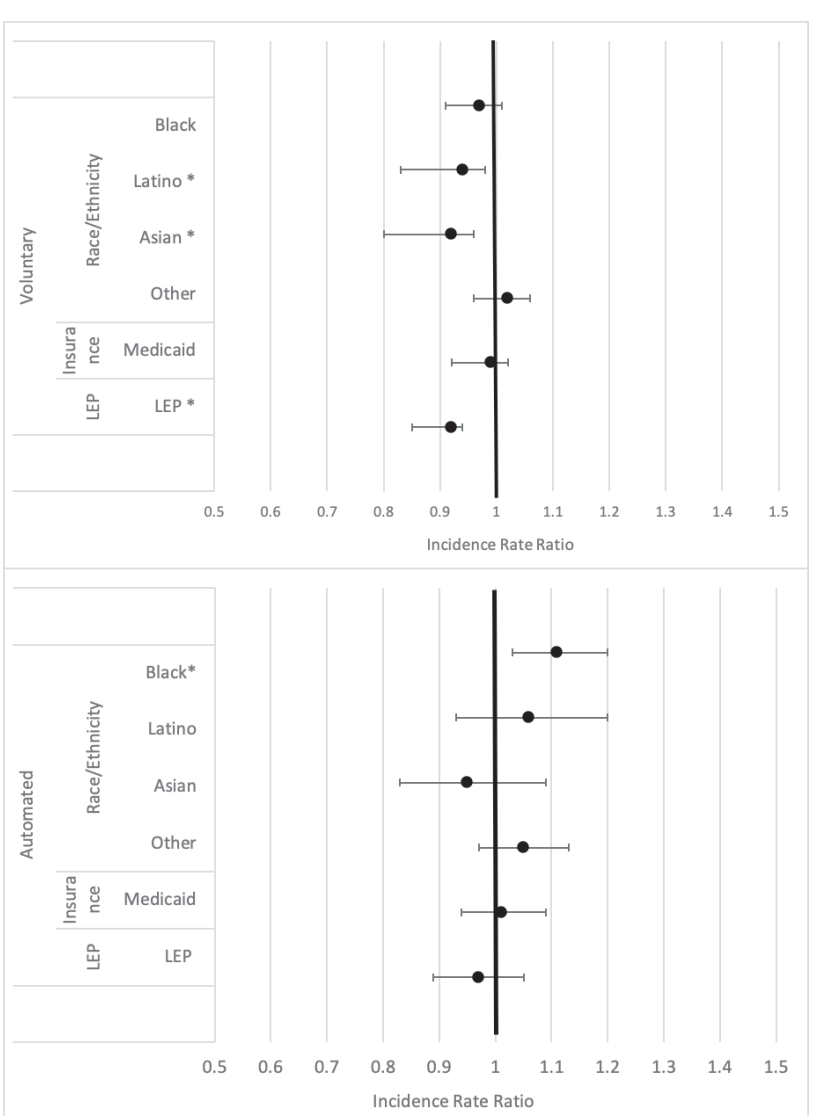

Figure 2 Adjusted analyses of risk for voluntary reported and automated events. LEP, limited English proficient; IRR, incident rate ratio. Adjusting for age, sex, marital status, primary diagnosis, clinical risk score, service at discharge, intensive care stay, length of stay and year of admission. Generalised estimation equations included to account for clustering for multiple admissions per patient. Race/Ethnicity, insurance and LEP are entered individually. $\mathrm{TP}<0.05$.

event (IRR 0.90, 95\% CI 0.82 to 0.99). There were no significant differences by language for automated events (online supplemental appendix table 3 ).

\section{DISCUSSION}

In this single-centre retrospective study of inpatient patient safety events, we found generally that the vulnerable populations of interest were not at increased risk of the patient safety events we examined. These results, however, obscure important findings that become apparent when examining events after stratifying by those that are detected through a voluntary reporting system vs automatically reported. We found that some racial and ethnic minorities (in particular Black patients) were experiencing more patient safety events, but only if the events were tracked using an automated method. These same groups appeared to have less frequent safety events when only voluntary reported system was used, suggesting possible underreporting. We saw similar patterns of events among patients with LEP, although Medicaid insurance did not seem to impact risk for voluntary reported or automated patient safety events, despite Medicaid insurance being more common in the populations under study.

There are at least two potential explanations for our findings. First, because these two systems are capturing different types of events, it is possible that vulnerable patients may in fact be more likely to experience patient safety events captured by automated systems but less likely to experience events captured by voluntary reported systems. Our findings that automated events, many of which are medication and infection-related errors, were more common for Black patients is consistent with some prior research using chart and billing data that demonstrated increased risk for hospital acquired infections and medicationrelated patient safety events for minority patients. ${ }^{19} 20$ In contrast, however, the types of events that previously have been shown to be higher in vulnerable populations are events that should be picked up by the voluntary reported event system, where we found lower risks for several of these vulnerable populations. For example, prior research using billing data has demonstrated increased risk of obstetric complications in racial/ethnic minorities and those with LEP. $^{21-24}$ Additionally, it is likely that LEP patients would be at increased risk for communication sensitive events compared with English patients due to communication barriers and such communication sensitive events also would more likely be captured in a voluntary event monitoring system rather than the automated system. Therefore, although it is possible that different populations of patients are at increased risk for different types of events, this does not seem to explain the discrepancy we found between voluntary reported and automated events.

The second and more likely explanation is that our findings are due, at least in part, to systematic differences in reporting of voluntary reported events. Our findings are consistent with prior research that suggests that safety events in general are under-reported when using a voluntary system, but our study also suggests that there may be more under-reporting in some populations compared with others, which has not been as well described. ${ }^{9-12}$ One study by Thomas et al found that a voluntary reported patient safety event system identified a higher proportion of patient safety events in White patients than that of other race/ethnicities. Unlike in our study, Thomas et al did not compare this system with another to identify patient safety events, but the authors suspected that this finding may have represented under-reporting of events among minority patients. ${ }^{25}$ There are several possible explanations for such under-reporting. Safety events may be more visible to providers if they occur in a White, English speaking patient. Due to factors beyond the healthcare system, these patients may be more likely to have the social capital to advocate for themselves and the health literacy to bring more subtle patient safety events to the attention of their providers. Additionally, these 
findings may be another example of bias in healthcare, and a voluntary reported system for patient safety events may therefore perpetuate those biases. In contrast, when automated tools are used to identify patient safety events, they may be less likely to perpetuate bias. Importantly, our results suggest that some patient groups might be at higher risk of safety events when just considering automatically detected events that likely remove any inherent bias in reporting that might arise with voluntary systems. However, the limitations of automated tools, namely that they are a crude measure of patient safety events and are unable to capture richer data in the way that voluntary systems do, also hinders their use.

While we can only speculate as to why we found a discrepancy in risk for patient safety events depending on method used to identify an event, our findings have important implications for healthcare systems. If systems are relying primarily on provider reporting systems to identify patient safety events and in turn develop new systems to address those events, then these interventions may not fully address the needs of vulnerable populations. This will result in lack of recognition of events and may disproportionally impact these more vulnerable populations, which, in turn, could exacerbate existing health disparities. ${ }^{25}$ There are strengths in voluntary reporting systems compared with automated reporting systems, but it is important for health systems to be mindful of the shortcomings of voluntary systems and make concerted efforts to address these shortcomings. In particular, given the current sociopolitical climate, many healthcare systems are re-enforcing their efforts to address bias and structural racism in order to improve health equity. Addressing patient safety is critical to this work. Our findings highlight the importance of examining patient safety events through the lens of race/ethnicity, insurance and LEP to assure that such under-reporting does not occur. ${ }^{26}$ Therefore, it is important to develop systems of identifying events and solutions to improving patient safety that reflect the complex interaction between patient safety and health disparities.

There are several limitations to our work. First, our study lacks generalisability to other institutions with different populations. Additionally, despite our comprehensive approach to identifying patient safety events, we certainly are missing some patient safety events that may have clinical relevance to both providers and patients. Additionally, as evidenced by our findings, our study still likely suffers from underreporting in events identified by the voluntary provider reporting system. Another important limitation is that the two systems we compare capture different domains and therefore there are some limits to an exact comparison. Nonetheless, prior research suggests that these subpopulations are at increased risk of events regardless of the domain used. Finally, although the automated system for identifying patient safety events was quite sensitive and specific, we may have identified hospital events that are not patient safety events. Additionally, some of the events captured in the voluntary system may not ultimately meet the criteria of a patient safety event. However, we would not expect to see differential event rates by race/ethnicity, insurance and LEP even if this were the case.

In conclusion, we found differences in risks of experiencing a patient safety event differed depending on the method used to identify events. Our findings suggest that voluntary reporting systems for patient safety events may be not effectively identify patient safety events in particularly vulnerable populations and, based on the rates of automated events, suggest that some vulnerable populations might be at increased risk of experiencing safety events.

\section{Author affiliations}

${ }^{1}$ General Internal Medicine, Boston Medical Center, Boston, MA, USA

${ }^{2}$ The RAND Corportation, Boston, MA, USA

${ }^{3}$ Center for Healthcare Delivery Science, Beth Israel Deaconess Medical Center, Boston, MA, USA

${ }^{4}$ Clinical Research Center, Soroka University Medical Center and Faculty of Health Sciences, Ben-Gurion University of the Negev, Beer-Sheva, Israel

${ }^{5}$ Health Care Quality, Beth Israel Deaconess Medical Center, Boston, MA, USA

${ }^{6}$ Division of General Medicine and Primary Care, Beth Israel Deaconess Medical Center, Boston, MA, USA

${ }^{7}$ Division for Pulmonary, Critical Care, and Sleep Medicine, Beth Israel Deaconess Medical Center, Boston, MA, USA

${ }^{8}$ Department of Health Care Policy, Harvard Medical School, Boston, MA, USA

Acknowledgements The authors would like to thank InSIGHT Core in the Center for Healthcare Delivery Science for assistance in obtaining primary data and programming assistance.

Contributors Conception: all authors. Data acquisition: LS, JPS. Data analysis and interpretation: LS, VN and BL. Drafting the article: LS and BL. Critical revision of the article: all authors. Final approval of the version to be published: all authors.

Funding The authors have not declared a specific grant for this research from any funding agency in the public, commercial or not-for-profit sectors.

Competing interests None declared.

Patient consent for publication Not required.

Ethics approval This study was approved by the Institutional Review Board at Beth Israel Deaconess Medical Center.

Provenance and peer review Not commissioned; externally peer reviewed.

Data availability statement No data are available.

Supplemental material This content has been supplied by the author(s). It has not been vetted by BMJ Publishing Group Limited (BMJ) and may not have been peer-reviewed. Any opinions or recommendations discussed are solely those of the author(s) and are not endorsed by BMJ. BMJ disclaims all liability and responsibility arising from any reliance placed on the content. Where the content includes any translated material, BMJ does not warrant the accuracy and reliability of the translations (including but not limited to local regulations, clinical guidelines, terminology, drug names and drug dosages), and is not responsible for any error and/or omissions arising from translation and adaptation or otherwise.

\section{ORCID ID}

Lucy B Schulson http://orcid.org/0000-0002-9774-6372 


\section{REFERENCES}

1 The Joint Commission. Comprehensive accreditation manual for hospitals 20162016.

2 Harris LM, Dreyer BP, Mendelsohn AL, et al. Liquid medication dosing errors by Hispanic parents: role of health literacy and English proficiency. Acad Pediatr 2017;17:403-10.

3 Weiss BD. Health Literacy and Patient Safety: Help Patients Understand. In: Manual for clinicians. 2nd edn. American Medical Association Foundation, 2007.

4 Sudore RL, Yaffe K, Satterfield S, et al. Limited literacy and mortality in the elderly: the health, aging, and body composition study. J Gen Intern Med 2006;21:806-12.

5 Tang AS, Kruger JF, Quan J, et al. From admission to discharge: patterns of interpreter use among resident physicians caring for hospitalized patients with limited English proficiency. J Health Care Poor Underserved 2014;25:1784-98.

6 Mayo R, Parker VG, Sherrill WW, et al. Cutting corners: provider perceptions of interpretation services and factors related to use of an AD hoc interpreter. Hisp Health Care Int 2016;14:73-80.

7 Wasserman M, Renfrew MR, Green AR, et al. Identifying and preventing medical errors in patients with limited English proficiency: key findings and tools for the field. J Healthc Qual 2014;36:5-16.

8 Institute of Medicine (US) Committee on Understanding and Eliminating Racial and Ethnic Disparities in Health Care. Unequal treatment: confronting racial and ethnic disparities in health care. Washington (DC): National Academies Press (US), 2003. http://www.ncbi.nlm.nih.gov/books/ NBK220358/

9 Classen DC, Resar R, Griffin F, et al. 'Global trigger tool' shows that adverse events in hospitals may be ten times greater than previously measured. Health Aff 2011;30:581-9.

10 Aspden P. Patient safety: achieving a new standard for care. Washington, D.C: National Academies Press, 2004. http:// public.eblib.com/choice/publicfullrecord.aspx? $\mathrm{p}=3376726$

11 Weissman JS, Schneider EC, Weingart SN, et al. Comparing patient-reported Hospital adverse events with medical record review: do patients know something that hospitals do not? Ann Intern Med 2008;149:100-8.

12 Weingart SN, Pagovich O, Sands DZ, et al. What can hospitalized patients tell us about adverse events? learning from patient-reported incidents. J Gen Intern Med $2005 ; 20: 830-6$.
13 Stockwell DC, Landrigan CP, Toomey SL, et al. Racial, ethnic, and socioeconomic disparities in patient safety events for hospitalized children. Hosp Pediatr 2019;9:1-5.

14 Griffin F, Resar R. IHI global trigger tool for measuring adverse events. IHI innovation series white paper. 2nd edn. Cambridge, MA: Institute for Healthcare Improvement, 2009. https:// www.rldatix.com/en-nam/products/risk

15 Rl Datix. Available: https://www.rldatix.com/en-nam/products/ risk

$16 \mathrm{Hu}$ Y. A quantitative approach to patient risk assessment and safety optimization in intensive care units 2017.

17 Healthcare Cost and Utilization Project (HCUP). Clinical classifications software (CCS) for ICD-10-PCS (beta version)., 2019. Available: www.hcup-us.ahrq.gov/toolssoftware/ccs10/ ccs10.jsp

18 Elixhauser A, Steiner C, Harris DR, et al. Comorbidity measures for use with administrative data. Med Care 1998;36:8-27.

19 Bakullari A, Metersky ML, Wang Y, et al. Racial and ethnic disparities in healthcare-associated infections in the United States, 2009-2011. Infect Control Hosp Epidemiol 2014;35:S10-16.

20 Metersky ML, Hunt DR, Kliman R, et al. Racial disparities in the frequency of patient safety events: results from the National Medicare patient safety monitoring system. Med Care 2011;49:504-10.

21 Hines AL, Andrews RM, Moy E, et al. Disparities in rates of inpatient mortality and adverse events: race/ethnicity and language as independent contributors. Int J Environ Res Public Health 2014;11:13017-34.

22 Howell EA, Zeitlin J, Hebert P, et al. Paradoxical trends and racial differences in obstetric quality and neonatal and maternal mortality. Obstet Gynecol 2013;121:1201-8.

23 Sentell T, Chang A, Ahn HJ, et al. Maternal language and adverse birth outcomes in a statewide analysis. Women Health 2016;56:257-80.

24 Grobman WA, Bailit JL, Rice MM, et al. Racial and ethnic disparities in maternal morbidity and obstetric care. Obstet Gynecol 2015;125:1460-7.

25 Thomas AD, Pandit C, Krevat SA. Race differences in reported harmful patient safety events in healthcare system high reliability organizations. J Patient Saf 2018. doi:10.1097/ PTS.0000000000000563. [Epub ahead of print: 21 Dec 2018].

26 Sivashanker K, Gandhi TK. Advancing safety and equity together. N Engl J Med 2020;382:301-3. 